\title{
Role of point of care ultrasound in COVID-19 pandemic: what lies beyond the horizon?
}

\author{
Felice Galluccio ${ }^{1}$, Tolga Ergonenc ${ }^{2}$, Ece Yamak Altinpulluk ${ }^{3,4}$, Maria Perez Herrero ${ }^{5}$, Maria \\ del Mar De la Torre Carpente ${ }^{6}$, Marco Capassoni ${ }^{1}$, Karla Espinoza ${ }^{7}$, Cristian Aragon Benedi ${ }^{8}$, \\ Abdallah El-Sayed Allam 9 , Vanni Orzalesi ${ }^{10}$, Mario Fajardo Perez ${ }^{8}$
}

\begin{abstract}
${ }^{1}$ Division of Rheumatology, Department of Clinical and Experimental Medicine, University of Florence, Italy, ${ }^{2}$ Department of Anesthesia and Reanimation, Sakarya University Education and Research Hospital, Sakarya, Turkey, ${ }^{3}$ Department of Outcomes Research, Anesthesiology Institute, Cleveland Clinic Foundation, Cleveland, Ohio, USA, ${ }^{4}$ Anesthesiology and Reanimation, Istanbul Universitesi-Cerrahpasa, Cerrahpasa Medical School, Istanbul, Turkey, ${ }^{5}$ Department of Anaesthesia and Reanimation, Hospital Clinico Universitario, Valladolid, Spain, ${ }^{6}$ Cardiology Department, Cardiovascular Imaging. Hospital Universitario Río Hortega, Valladolid, Spain, ${ }^{7}$ Anesthesia, Hospital Mexico Costa Rica, San Jose, Costa Rica, ${ }^{8}$ Department of Anesthesia and Reanimation, Hospital Universitario Mostoles, Madrid, Spain, ${ }^{9}$ Department of Physical Medicine, Rheumatology and Rehabilitation, Tanta University Hospitals \& Faculty of Medicine, Tanta University, Egypt, ${ }^{10}$ Neuroanesthesia and Resuscitation Unit, University Hospital AOU Careggi, Florence, Italy
\end{abstract}

\begin{abstract}
The pandemic of COVID-19 requires rapid and easy access to reliable imaging modalities for diagnosis and follow up. Considering the cost-effectiveness of the imaging used, ultrasound is a non-ionizing, portable and bedside imaging modality with a high diagnostic impact in emergencies and intensive care units in pandemics, but it is operator dependent. In our article, we provide a comprehensive review of the role of point-of-care ultrasound in the diagnosis of COVID-19 infection and its impact on the lungs, cardiovascular system, eyes and abdominal organs. Moreover, ultrasound can provide real-time diagnostic and therapeutic interventions, such as the placement of a central catheter and aspiration of pericardial effusion. Awareness of health care professionals in the front-line fighting COVID-19 infection in emergency rooms, clinics, and in intensive care units is important and will help rapid and targeted management decisions.
\end{abstract}

Keywords: POCUS; coronavirus; COVID-19; pandemic; ultrasound

\section{Introduction}

The Sars-CoV-2 pandemic, with many millions of confirmed cases and hundreds of thousands of deaths in 185 countries around the world [1], is a medical emergency we have never faced. The pandemic outbreak has

Received 08.05.2020 Accepted 24.06.2020

Med Ultrason

2020, Vol. 22, No 4, 461-468

Corresponding author: Felice Galluccio MD

Fisiotech Lab, Via Giovanni Lanza 56, 50136 Firenze (FI), Italia

Email: felicegalluccio@gmail.com

ORCID: https://orcid.org/0000-0001-7485-471X led to an overwhelming number of severe cases, which has compromised the delivery of health services, with difficulties in guaranteeing diagnostic tests, imaging, ventilators, health personnel, and personal protection equipment [1-3]. Although chest x-ray and high-resolution computed tomography (HRCT) are the gold standards for diagnosis and follow-up of pneumonia, in these conditions at a high risk of contamination of imaging devices, hospital environments, and with resource constraints, their applicability is limited and difficult to manage [4]. Moreover, critically ill patients are at a high risk of destabilization during the transport to the radiology units; therefore, bedside imaging may play a fundamental role. Some patients develop severe forms with adult 
respiratory distress syndrome (ARDS) and multi-organ dysfunction including myocardial involvement (myocarditis, pericarditis, infarction, etc), heart failure, acute kidney failure, liver and spleen dysfunction, disseminated intravascular coagulation or thromboembolism, viral and necrotizing encephalitis and brain edema [5-12].

In this context, ultrasound (US) is the method that best meets these needs. Focused or point-of-care US (POCUS) is defined as ultrasonography brought to the bedside for real-time performance [13] and can be employed out of hospitals for emergency or disaster triage [14-16]. Images are collected immediately and are easily repeatable in cases when the patient's condition changes. POCUS is performed in different medical specialties for vastly different clinical conditions both for diagnostic, procedural, and screening purposes. Many hospitals had to reallocate healthcare workers to cope with the COVID-19 pandemic. Several medical specialists, such as emergency physicians, intensivists and anaesthesiologists, internists, cardiologists, rheumatologists and pain therapists, adopted POCUS in their clinical practice both as a diagnostic and interventional tool and could be employed, as a multidisciplinary unit, in the management of COVID-19. The purpose of this review is to evaluate all the fields of application of POCUS ultrasound in COVID-19, based on literature and our clinical experience.

\section{General recommendation on safety and protection}

In the setting of COVID-19, the risk of contamination and infection is extremely high, so the most important thing is to protect the operator, assistant staff, and patients. Consider having a dedicated ultrasound machine just for COVID-19 patients so as not to transmit the disease to staff or other patients. Our suggestion is to disinfect first the probe(s), cables, and machine with wipes, then apply the first cover to the probe(s) and secure with elastic bands, then also cover the ultrasound machine.
Before entering the room and/or scan patients, put a second cover. In this way, you change from patient to patient the external cover and disinfect the internal one, in order also to protect the probe from chemical damage. Always wear appropriate personal protective equipment (mask, face shield, gown, double gloves etc.).

\section{Lung ultrasound}

For lung US is possible to use a micro-convex or convex array, phased array, and linear probes. Convex and linear probes allow better resolution of superficial pathology (subpleural consolidation) than a phased array. Linear probes are suggested to be used in early or mild disease to provide rich information of minor pleural or subpleural lesions, thus improving accuracy. Although it is possible to use both probes (linear + convex) to complete the examination, it requires more time, therefore, a higher risk of contamination. We recommend a 12-point lung scan because the disease distribution is patchy and may involve any part of the lung, as reported in CT studies [17]. Patient positioning may vary depending on disease severity. For early to moderate disease patients can be upright or in the lateral position with the arm abducted. In supine decubitus it is possible to lateral roll the patient to access the posterior lung where in prone the swimmer position [18] can help to scan the anterior lung. The following patterns have been described in COVID-19 patients: localized or diffused (fig 1A) and confluent B-lines (fig 1B) with spare areas, irregular and thickened pleural line with scattered discontinuities (fig 1C), subpleural and alveolar consolidations (tissue-like with air bronchograms) (fig 1D). Pleural effusion is rare while localized effusion around the lesions are detectable and related to crazy paving CT images (fig 1E) [19,20]. The disease can gradually develop, or suddenly deteriorate into severe and critically ill. Focal B lines are the main feature of early-stage and mild disease, while alveolar consolidation is the main feature of the progres-

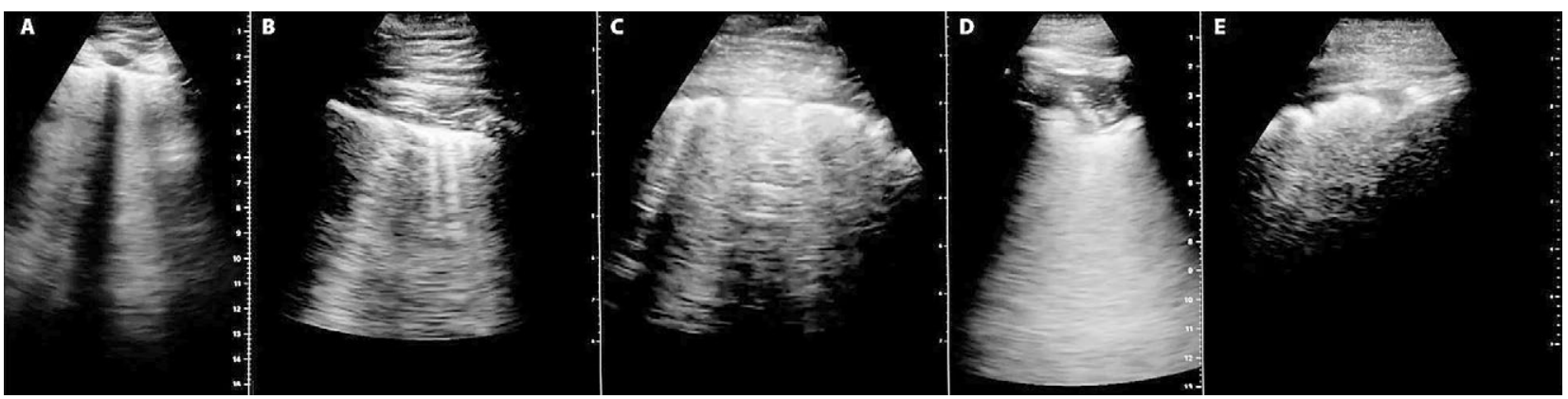

Fig 1. Lung ultrasound: A) B-lines; B) confluent B-lines; C) pleural line with scattered discontinuities; D) consolidation with air bronchograms; E) Localized pleural effusion related to crazy-paving on CT scans. 
sive stage and in severe disease. Prompt clinical identification, early assessment, and rapid severe grading are crucial to improving prognosis. A lines can be found in convalescent, and thickened pleural line with irregular B lines can be found in lung fibrosis [19]. Although it is not yet a proven and widespread methodology, lung elastography [21-23] could be a valuable aid in differentiating the stages of lung disease in covid-19 [24].

\section{Cardiac ultrasound}

Focused cardiovascular US aims to confirm clinical findings and is complementary to clinical and laboratory findings. Standard 2D B Mode scanning for a qualitative "gross visual assessment" is what we need in the clinical setting to manage patients. Based on cardiac pathology described in COVID-19, we recommend performing four standard cameras to look for suspicious sign for ST-Elevation Myocardial Infarction (STEMI), pulmonary embolism (PE), myocarditis and pericarditis or tamponade $[6,12]$.

It is recommended to use a cardiac phased array probe; alternatively, a convex can be used. For the parasternal long-axis view (PLAX) (fig 2A), the beam must be oriented with the long axis of the left ventricle. The transducer is placed on the $3^{\text {rd }}-5^{\text {th }}$ intercostal space at the left of the sternum with the marker orientated to the right clavicle. In this view, it is possible to examine the pericardial space (PS) for effusion (fig 2B), dimensions and contractility of left and right ventricles (LV and RV) and septum, movement of mitral valve (MV) anterior leaflet, of aortic root, and left atrium (LA). Rotating the probe clockwise 90 degrees, the parasternal short-axis view (PSAX) is obtained. Tilting the probe, we can examine at the level of papillary muscles, MV, and aortic valve (AV). Assess for regional abnormal wall motion, PS effusion, and the relative shapes and sizes of the two ventricles (LV round-shaped and RV crescent-shaped) (fig $2 \mathrm{C}$ with D-sign). To obtain the apical four-chamber view (A4C), put the probe at the point of the maximum impulse (or $5^{\text {th }}$ intercostal space near the anterior axillary line) with the beam directed to the patient head with the marker at 3 o'clock (fig 2D). By rotating the probe 45-90 degrees anticlockwise, we obtain the apical two chambers view (A2C) useful to visualize the true anterior and true inferior walls of the $\mathrm{LV}$, which is important for the assessment of regional wall motion abnormalities. The subcostal long-axis view (SLAX) may provide the only achievable view in technically challenging patients, such as those who are receiving mechanical ventilation or with chronic pulmonary diseases. For obtaining this view, the probe is put below and slightly to the right of the xiphi-

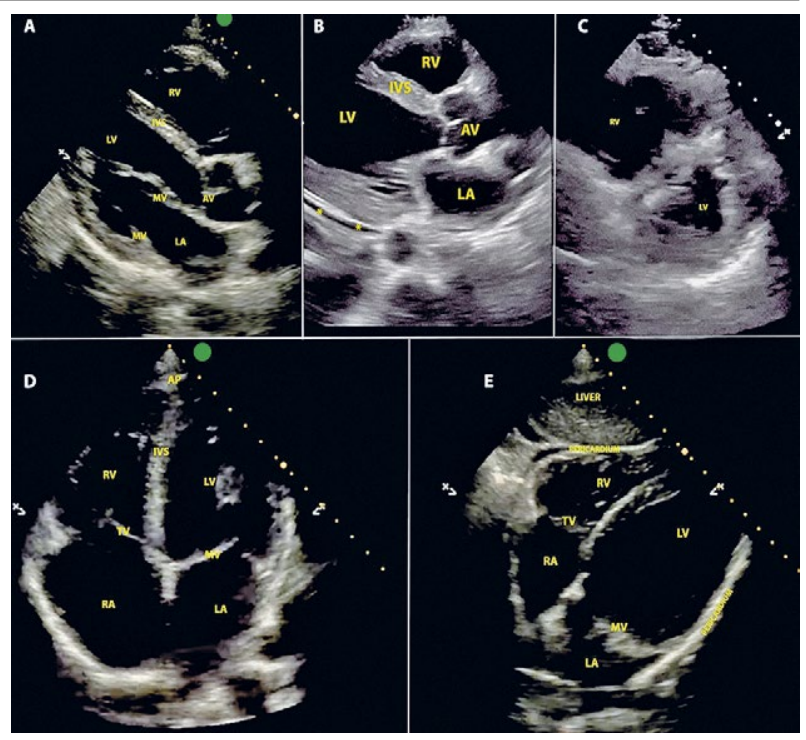

Fig 2. Cardiac ultrasound: A) parasternal long-axis view; B) parasternal long-axis view with pericardial effusion $(*)$; C) parasternal short-axis view with D-sign; D) apical fourchamber view; E) subcostal long-axis view.

sternum with the marker at 3 o'clock position and tilting anteriorly. It may be necessary to push into the abdomen to achieve this view. Examine PS, LV, RV, LA, RA, and up and down movement of MV (LV function) (fig 2E). By moving the probe, it is possible also to evaluate the inferior vena cava (IVC).

The most common findings are the regional abnormal wall motion in typical segments of coronary artery distribution in STEMI; the change of shape of RV with the flattened interventricular septum and RV/LV ratio $>1$ and dilated and non-collapsible IVC in PE; pericardial effusion and thickened pericardium in pericarditis; regional or global LV dysfunction and LV dilation or thickening of the ventricular wall due to edema in myocarditis and pericardial effusion with RV and RA collapse with dilated and non-collapsible IVC in tamponade.

\section{Vascular ultrasound}

POCUS vascular ultrasound focuses on congestive status and thrombosis assessment. For congestion, hepatic, portal vein, intra-renal venous Doppler must be evaluated with the IVC. Severe venous congestion was defined as the presence of severe flow abnormalities in multiple Doppler patterns with a dilated IVC $(\geq 2 \mathrm{~cm})$. The complete method for hepatic, portal, and renal Doppler assessment has been previously published [25]. For the hepatic vein Doppler, a systolic phase was of lesser amplitude than the diastolic phase, but towards the liver was considered mild while the presence of a reversed 
systolic phase was considered severe [26]. For the portal vein Doppler, a pulsatility fraction (PF) of $30-49 \%$ was considered mild while a $\mathrm{PF}>50 \%$ was considered severe. The IVC diameter was measured in its intra-hepatic portion at $2 \mathrm{~cm}$ of the junction with the hepatic veins using a longitudinal view from a sub-xiphoid position [27]. When the sub-xiphoid window was not appropriate, the probe was moved laterally to the right side of the body, over the liver, until an adequate view was achieved. For thrombosis, we recommend a 3-point lower leg examination with a linear probe at the common femoral vein, superficial femoral vein, and popliteal vein. For obtaining a better view, the lower extremity is put in a frog-like position, and the scan starts from proximal to distal using compression to evaluate the vein collapse (fig 3A,B). For routine scans, it is not necessary color-Doppler [28] nor augmentation (calf squeeze) because of the high incidence of thrombosis in COVID-19 patients. Noncollapsible vein, direct clot visualization (fig 3C), or no color-Doppler flow with augmentation (fig 3D) are signs of vein thrombosis. Jugular and subclavian veins should also be included in a routine examination.

\section{Abdominal ultrasound}

In COVID-19 the use of FAST scanning protocol [29] can be added to a routine evaluation in COVID clinics to identify early signs of multi-organ failure or suggestive of secondary hemophagocytic lymphohistiocytosis (sHLH). Splenomegaly was the most common finding followed by hepatomegaly with increased or heterogeneous echogenicity, ascites, gallbladder wall thickening, periportal echogenicity, lymphadenopathy and pleural effusion [30,31]. Chateil et al speculated that the increased periportal echogenicity and gallbladder wall thickening seen in many patients with HLH could represent the portal lymphohistiocytic infiltration and lymphatic and/or portal venous stasis [32]. Regression or disappearance after treatment has also been reported, suggesting a potential role in the monitoring of the disease.

With the right and left upper quadrants and an anterior scan, we can evaluate the presence of intraperitoneal fluid, dimensions, and echogenicity of the spleen and liver, and to check the gallbladder walls and periportal echogenicity. In the supine position, start in the left upper quadrant near the posterior axillary line in the area of the 10th rib to identify the spleen in the longitudinal view. Align the probe with the long axis of the spleen to measure (fig 4A). Splenomegaly was defined as an anteroposterior dimension $>13 \mathrm{~cm}$ [33]. To evaluate echogenicity and check for fluid around the spleen or in between the spleen and left kidney, the entire spleen

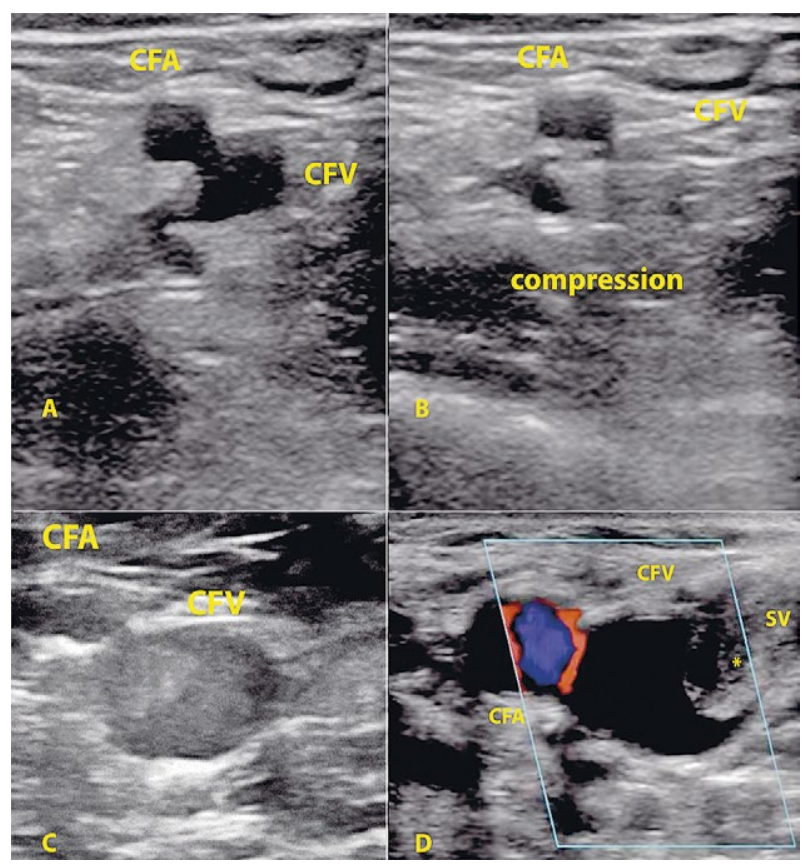

Fig 3. Vascular ultrasound: $A)$ common femoral vein (CFV) scan in frog-like position; B) under compression; C) direct clot visualization; D) direct clot visualization $(*)$ in the saphenous vein $(\mathrm{SV})$ and $\mathrm{CFV}$ with no Color-Doppler flow with augmentation technique (calf compression).

should be scanned from the upper to the lower pole. The gallbladder wall thickening $(>3 \mathrm{~mm})$ and the periportal echogenicity are evaluated in the anterior view near the midclavicular line, then the probe is moved to the right upper quadrant to scan the Morison pouch and to measure craniocaudally the right liver lobe for hepatomegaly $(>15 \mathrm{~cm}$ ) (fig 4B) [34].

Considering that renal dysfunction and acute kidney injury (AKI) are not uncommon in patients hospitalized for COVID-19 and are associated with mortality [35], it is essential to scan the kidneys for assessing the kidney's

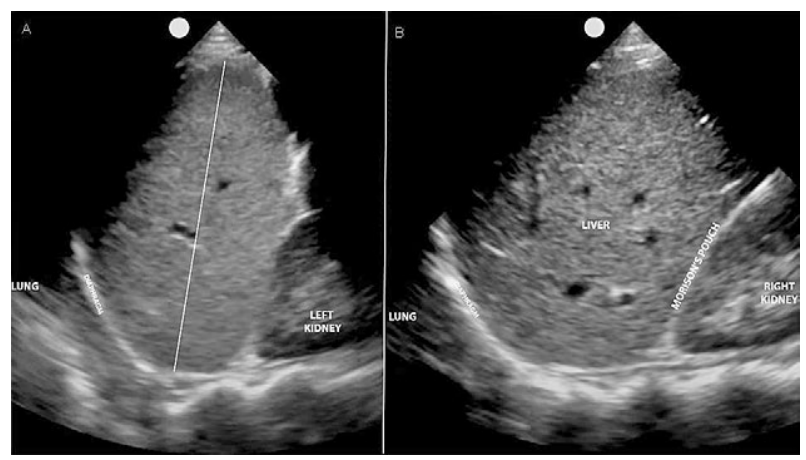

Fig 4. Abdominal ultrasound: A) Left upper quadrant scan with long axis spleen visualization; B) Right upper quadrant scan with right liver lobe, kidney and Morison pouch visualization. 


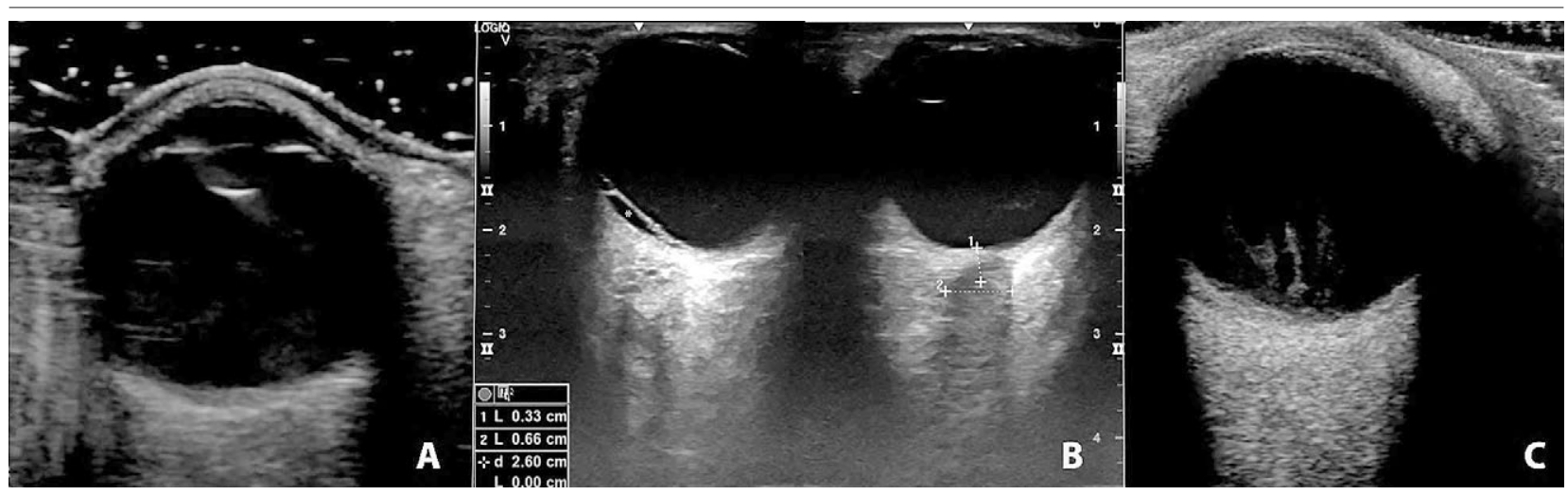

Fig 5. Ocular ultrasound: A) Eye transversal scan; B) Measurement of the optic nerve sheath suspected of intracranial hypertension $(0.66 \mathrm{~cm})$ and retinal detachment $(*)$; C) Vitreous haemorrhage.

size, echogenicity, and cortical and parenchymal thickness. Large kidneys in AKI (pole-to-pole 10-12 cm) may result from acute glomerulonephritis and acute interstitial nephritis (due to edema and inflammatory infiltrates) and renal vein thrombosis (edema from congestion) [36]. Increased cortical echogenicity (relative to liver or spleen) can be seen in AKI where inflammatory infiltrates and proteinaceous casts reflect sound waves, like in acute glomerulonephritis and acute tubular necrosis. Decreased cortical echogenicity usually results from interstitial edema in cases of inflammation or infection. The cortical thickness, measured from the base of the medullary pyramid to the outer edge of the kidney, is about $7-10 \mathrm{~mm}$, being thicker at the poles and is useful for distinguishing between AKI and chronic disease where it is generally reduced [37].

\section{Ocular ultrasound}

Ocular ultrasound is a relatively new application but its ability to evaluate the eye is also of great value in COVID-19 patients. This is a quick, precise and noninvasive tool to evaluate various conditions such as vitreous haemorrhage, retinal thrombosis, papilledema due to increased intracranial pressure, as for central venous thrombosis, inflammatory diseases, etc. [38-43]. A linear probe (7.5-10 MHz or more) is used to scan with a closed eye technique. Gel stand-off technique is useful to not compress the eye. The eye can be scanned both in sagittal and transversal planes. The depth must be adjusted to allow visualization of the optic nerve posterior to the eyeball. The structures of the eye are easily visible, since it is a globe filled with liquid (fig 5A). The anterior, posterior and vitreous chambers are anechoic and divided from each other by clearly recognizable hyperechoic structures. The normal retina, on the other hand, cannot be distinguished from the other choroidal layers.
Behind the eyeball, we recognize the optic nerve as a linear hypoechoic structure that moves away posteriorly. The evaluation of the diameter of the optic nerve sheath is a simple non-invasive procedure that provides us with an evaluation of intracranial pressure [43]. A normal optic nerve sheath measures up to $5.0 \mathrm{~mm}$ in diameter at $3 \mathrm{~mm}$ posterior to the globe. A normal optic nerve sheath measures up to $5.0 \mathrm{~mm}$ in diameter at $3 \mathrm{~mm}$ posterior to the globe. An average size between 5 and 6 or more than $6 \mathrm{~mm}$ is considered abnormal and elevated intracranial pressure should be suspected (fig 5B). It is recommended not to take the measurement in the pronated patient and to wait at least an hour after supination. The position in pronation, either with straight or rotated head, could increase the stasis resulting in an overestimated measure. Vitreous haemorrhage appears as echogenic material in the posterior chamber (fig 5C). Retinal thrombosis appears as a dilated optic nerve sheath with hyperechoic material (clot).

\section{Ultrasound guided interventions}

Patients with COVID-19, especially those in ICUs, require interventional procedures such as central venous catheter placement and arterial cannulation.

Central venous catheter placement is mainly performed in the internal jugular vein (IJV) or the subclavian vein (SV), in three consecutive steps. The first step is the needle, guidewire, and catheter insertion into the vein with the landmark technique. In the second step, a chest $\mathrm{x}$-ray is performed to check its positioning (catheter tip placement or mispositioning). The third step is to monitor the possible adverse events, such as pneumothorax. These steps can be performed under US guidance, avoiding the transfer of the patient, thereby reducing the risk of contamination or destabilization. Furthermore, with the landmark technique, the complication rate ranges from 
$0.3 \%$ to $18.8 \%$ (catheter malposition, arterial puncture, hematoma, air embolism, nerve injury, pneumo- and/or hemothorax) $[44,45]$. US guided central venous catheter (CVC) procedures have a significantly lower failure rate as compared to the landmark technique and are associated with a decreased rate of complications due to shorter access time and fewer attempts $[46,47]$ which is essential in COVID-19 patients.

Currently, it is recommended that needle insertion and placement, the first and second step of CVC procedures, be performed under real-time US guidance without x-ray. Catheter tip optimal position is performed by directly visualizing the right atrium and superior vena cava. Moreover, lung US can detect a pneumothorax more accurately than an x-ray. Third, lung ultrasonography has a sensitivity of $95.3 \%$ and a specificity of $91.1 \%$ for the diagnosis of the pneumothorax in the ICU patients [48]. The early diagnosis and treatment of a pneumothorax is critical. Lung US allows a quick evaluation of the critically ill and hemodynamically unstable patient at the bedside. In the mid-clavicular line, the second to fourth intercostal spaces are the most suitable area for detecting pneumothoraxes in the supine patient [49,50], using a high frequency linear or a convex. The lack of lung sliding is one of the main B-mode US findings of pneumothorax. In M-mode, pneumothorax shows a typical pattern called "barcode sign", in contrast to the "sandon-the-beach" appearance of the normal lung. The gas interface creates a high reflective surface hiding not only the lung sliding but also the B-lines. On the contrary, A-lines show an increased clarity confirming the pneumothorax. Finally, US allows the evaluation of the mid/ long term complications of the $\mathrm{CVC}$, such as thrombosis, arteriovenous fistula, and pseudoaneurysm [51,52]. Bedside ultrasonography could become the new standard for the central venous catheter, the arterial line procedures and the control of complications. Finally, bedside ultrasound-guided percutaneous tracheostomy is a procedure that can be performed directly in ICUs. Compared to the bronchoscopy-guided procedure or traditional surgery, it allows to correctly visualize the anatomical structures of the neck, resulting in better safety and with less complications $[53,54]$.

\section{Conclusions}

During a pandemic, like COVID-19, we need extra precision and safety, so only necessary procedures should be performed to minimize the risk of exposure to the virus for patients and healthcare workers. In the last years, POCUS has been widespread, and more and more doctors have adopted it in their clinical practice, because it is easy and quick to complete, thus playing an essential role in the management of COVID-19 patients and the triage of suspicious cases. Although the US is not considered the gold standard for lung imaging, the benefits of it should not be neglected. The accuracy of lung US in detecting pneumonia and ARDS is well described as well for organ involvement and complications. One of the major advantages is that the same doctor visits and performs the US examination, thus limiting the personnel and devices used, with a marked reduction in the risk of infection. We strongly suggest implementing POCUS in the diagnosis and management of COVID-19 and integrating it directly into the medical decision-making process.

We also consider appropriate, in anticipation of future scenarios, to implement the teaching of point-of-care ultrasound to all personnel dedicated to emergency management.

\section{Conflict of interest: none}

\section{References}

1. The Lancet. COVID-19: protecting health-care workers. Lancet 2020;395:922.

2. Schwartz J,. King CC, Yen MY. Protecting Health Care Workers during the COVID-19 Coronavirus Outbreak -Lessons from Taiwan's SARS response. Clin Infect Dis 2020 Mar 12. doi:10.1093/cid/ciaa255.

3. Huh S. How to train the health personnel for protecting themselves from novel coronavirus (COVID-19) infection during their patient or suspected case care. J Educ Eval Health Prof 2020;17:10.

4. Buonsenso D, Pata D, Chiaretti A. COVID-19 outbreak: less stethoscope, more ultrasound. Lancet Respir Med 2020;8:e27.

5. Dong N, Cai J, Zhou Y, Liu J, Li F. End-stage Heart Failure with COVID-19: Strong Evidence of Myocardial Injury by 2019-nCoV. JACC Heart Fail 2020;8:515-517.

6. Mishra A K, Sahu K K, Lal A, Sargent J. Patterns of heart Injury in COVID - 19 and relation to outcome. J MedVirol 2020;10.1002/jmv.25847.

7. Feng G, Zheng K, Yan QQ, et al. COVID-19 and Liver Dysfunction: Current Insights and Emergent Therapeutic Strategies, J Clin Transl Hepatol 2020;8:18-24.

8. Cui S, Chen S, Li X, Liu S, Wang F. Prevalence of venous thromboembolism in patients with severe novel coronavirus pneumonia. J Thromb Haemost 2020;18:1421-1424.

9. Tang N, Li D, Wang X, Sun Z. Abnormal coagulation parameters are associated with poor prognosis in patients with novel coronavirus pneumonia. J Thromb Haemost 2020;18:844-847.

10. Poyiadji N, Shahin G, Noujaim D, Stone M, Patel S, Griffith B. COVID-19-associated Acute Hemorrhagic Necrotizing Encephalopathy: CT and MRI Features. Radiology 2020 Mar 31. doi:10.1148/radiol.2020201187. 
11. Wu Y, Xu X, Chen Z, et al. Nervous system involvement after infection with COVID-19 and other coronaviruses. Brain Behav Immun 2020;87:18-22.

12. Guo T, Fan Y, Chen M, et al. Cardiovascular Implications of Fatal Outcomes of Patients with Coronavirus Disease 2019 (COVID-19). JAMA Cardiol 2020 Mar 27. doi:10.1001/ jamacardio.2020.1017.

13. Moore CL, Copel JA. Point-of-care ultrasonography. N Engl J Med 2011;364: 749-757.

14. Nelson BP, Sanghvi A. Out of hospital point of care ultrasound: current use models and future directions. Eur J Trauma Emerg Surg 2016:42:139-150.

15. Wydo SM, Seamon MJ, Melanson SW, Thomas P, Bahner DP, Stawicki SP. Portable ultrasound in disaster triage: a focused review. Eur J Trauma Emerg Surg 2016;42:151-159.

16. Whitson MR, Mayo PH. Ultrasonography in the emergency department. Crit Care 2016;20:227.

17. Ye Z, Zhang Y, Wang Y, Huang Z, Song B. Chest CT manifestations of new coronavirus disease 2019 (COVID-19): a pictorial review. Eur Radiol. 2020 Mar 19. doi:10.1007/ s00330-020-06801-0.

18. Ugalde D, Medel JN, Romero C, Cornejo R. Transthoracic cardiac ultrasound in prone position: a technique variation description. Intensive Care Med 2018;44:986-987.

19. Peng Q Y, Wang XT, Zhang LN, Chinese Critical Care Ultrasound Study Group (CCUSG). Findings of lung ultrasonography of novel corona virus pneumonia during the 2019-2020 epidemic. Intensive Care Medicine 2020;46:849-850.

20. Huang Y, Wang S, Liu Y, et al. A Preliminary Study on the Ultrasonic Manifestations of Peripulmonary Lesions of Non-Critical Novel Coronavirus Pneumonia (COVID-19)," SSRN Electron J 2020 Feb 26. doi:10.2139/ssrn.3544750.

21. Clay R, Bartholmai BJ, Zhou B, et al. Assessment of Interstitial Lung Disease Using Lung Ultrasound Surface Wave Elastography: A Novel Technique With Clinicoradiologic Correlates. J Thorac Imaging 2019;34:313-319.

22. Zhou B, Zhang X. Lung mass density analysis using deep neural network and lung ultrasound surface wave elastography. Ultrasonics 2018;89:173-177.

23. Zhou B, Bartholmai BJ, Kalra S, Zhang X. Predicting lung mass density of patients with interstitial lung disease and healthy subjects using deep neural network and lung ultrasound surface wave elastography. J Mech Behav Biomed Mater 2020;104:103682.

24. Gattinoni L, Chiumello D, Caironi P, et al. COVID-19 pneumonia: different respiratory treatment for different phenotypes ? Intensive Care Med 2020;46:1099-1102.

25. Beaubien-Souligny W, Benkreira A, Robillard P, et al. Alterations in portal vein flow and intrarenal venous flow are associated with acute kidney injury after cardiac surgery: A prospective observational cohort study, J Am Heart Assoc 2018;7:e009961.

26. Rudski LG, Lai WW, Afilalo J, et al. Guidelines for the Echocardiographic Assessment of the Right Heart in Adults: A Report from the American Society of Echocardiography. Endorsed by the European Association of Echo- cardiography, a registered branch of the European Society of Cardiology, and the Canadian Society of Echocardiography. J Am Soc Echocardiogr 2010;23:685-713.

27. Wallace DJ, Allison M, Stone MB. Inferior vena cava percentage collapse during respiration is affected by the sampling location: An ultrasound study in healthy volunteers. Acad Emerg Med 2010;17:96-99.

28. Lensing AW, Doris CI, cGrath FP, et al. A comparison of compression ultrasound with color Doppler ultrasound for the diagnosis of symptomless postoperative deep vein thrombosis. Arch Intern Med 1997;157:765-768.

29. Scalea TM, Rodriguez A, Chiu WC, et al. Focused assessment with sonography for trauma (FAST): Results from an International Consensus Conference. J Trauma 1999;46:466-472.

30. Schmidt MH, Sung L, Shuckett BM. Hemophagocytic Lymphohistiocytosis in Children: Abdominal US Findings within 1 Week of Presentation," Radiology 2004;230:685-689.

31. Fitzgerald NE, McClain KL. Imaging characteristics of hemophagocytic lymphohistiocytosis. Pediatr Radiol 2003;33:392-401.

32. Chateil JF, Brun M, Perel Y, Pillet P, Micheau M, Diard F. Abdominal ultrasound findings in children with hemophagocytic lymphohistiocytosis. Eur Radiol 1999;9:474-477.

33. Olson APJ, Trappey B, Wagner M, Newman M, Nixon LJ, Schnobrich D. Point-of-care ultrasonography improves the diagnosis of splenomegaly in hospitalized patients. Crit Ultrasound J 2015;7:13.

34. Riestra-Candelaria BL, Rodriguez-Mojica W, Jorge JC. Anatomical criteria to measure the adult right liver lobe by ultrasound. Sonography 2018;5:181-186.

35. Cheng Y, Luo R, Wang K, et al. Kidney disease is associated with in-hospital death of patients with COVID-19. Kidney Int 2020;97:829-838.

36. Faubel S, Patel NU, Lockhart ME, Cadnapaphornchai MA. Renal relevant radiology: Use of ultrasonography in patients with AKI. Clin J Am Soc Nephrol 2014;9:382-394.

37. Kelahan LC, Desser TS, Troxell ML, Kamaya A. Ultrasound Assessment of Acute Kidney Injury. Ultrasound Q 2019 Jun;35(2):173-180.

38. Riccardi A, Siniscalchi C, Lerza R. Embolic Central Retinal Artery Occlusion Detected with Point-of-care Ultrasonography in the Emergency Department. J Emerg Med 2016;50:e183-e185.

39. Arthur J, Duran-Gehring P, Kumetz C, Chadwick S, McIntosh M. Cerebral Venous Thrombosis: An Uncommon Cause of Papilledema on Bedside Ocular Ultrasound. J Emerg Med 2019;56:288-293.

40. Teismann N, Lenaghan P, Nolan R, Stein J, Green A. Pointof-care Ocular Ultrasound to Detect Optic Disc Swelling. 2013;20:920-925.

41. Stoner-Duncan B, Morris S. Early Identification of Central Retinal Artery Occlusion Using Point-of-care Ultrasound. Clin Pract Cases Emerg Med 2019;3:13-15.

42. Kilker BA, Holst JM, Hoffmann B. Bedside ocular ultrasound in the emergency department. Eur J Emerg Med 2014;21:246-253. 
43. Blaivas M, Theodoro D, Sierzenski PR. Elevated Intracranial Pressure Detected by Bedside Emergency Ultrasonography of the Optic Nerve Sheath. Acad Emerg Med 2003;10:376-381.

44. Merrer J, De Jonghe B, Golliot F, et al. Complications of femoral and subclavian venous catheterization in critically III patients: A randomized controlled trial. J Am Med Assoc 2001;286:700-707.

45. Goldfarb G, Lebrec D. Percutaneous cannulation of the internal jugular vein in patients with coagulopathies: an experience based on 1,000 attempts. Anesthesiology 1982;56:321-323.

46. Wu SY, Ling Q, Cao LH, Wang J, Xu MX, Zeng WA. Real-time two-dimensional ultrasound guidance for central venous cannulation: A meta-analysis. Anesthesiology 2013;118:361-375.

47. Leung J, Duffy M, Finckh A. Real-Time Ultrasonographically-Guided Internal Jugular Vein Catheterization in the Emergency Department Increases Success Rates and Reduces Complications: A Randomized, Prospective Study. Ann Emerg Med 2006;48:540-547.

48. Lichtenstein DA, Menu Y. A bedside ultrasound sign ruling out pneumothorax in the critically ill: Lung sliding. Chest 1995;108:1345-1348.
49. Tocino IM, Miller MH, Fairfax WR. Distribution of pneumothorax in the supine and semirecumbent critically ill adult. Am J Roentgenol 1985;144:901-905.

50. Ball CG, Kirkpatrick AW, Laupland KB, et al. Factors related to the failure of radiographic recognition of occult posttraumatic pneumothoraces. Am J Surg 2005;189:541546.

51. Yilmaz KB, Akinci M, Dogan L, Yologlu Z, Atalay C, Kulacoglu H. Central venous catheter-associated thrombosis in the perioperative period: A frequent complication in cancer patients that can be detected early with Doppler examination. Tumori 2010;96:690694.

52. Ahmad F, Turner SA, Torrie P, Gibson M. Iatrogenic femoral artery pseudoaneurysms - A review of current methods of diagnosis and treatment. Clin Radiol 2008;63:13101316.

53. Ravi PR, Vijai MN, an Shouche S. Realtime ultrasound guided percutaneous tracheostomy in emergency setting: the glass ceiling has been broken. Disaster Mil Med 2017;3:7.

54. Guinot PG, Zogheib E, Petiot S, et al. Ultrasound-guided percutaneous tracheostomy in critically ill obese patients. Crit Care 2012;16:R40. 